\section{Punção aspirativa por agulha fina em tumores cervicais}

\author{
Rafael R. Malinsky', Daniela P. Dall'Igna ${ }^{2}$, \\ Mariana M. Smith ${ }^{3}$, Sady S. da Costa ${ }^{4}$
}

Fine-needle aspiration biopsy in neck luimps

Palavras-chave: tumor cervical, punção aspirativa por agulha fina, citologia.

Key words: cervical tumor, puncture aspirativa for fine needle, cytology.

\section{Resumo / Summary}

\section{I}

ntrodução: Inúmeras são as patologias que podem se apresentar inicialmente como uma lesão em região cervical, o que torna o seu diagnóstico nem sempre simples. A punção aspirativa por agulha fina (PAAF) é um método simples, seguro e eficaz no sentido de auxiliar o diagnóstico de tumorações cervicais. Objetivo: Avaliar o desempenho diagnóstico da PAAF em lesões cervicais realizadas no Serviço de Otorrinolaringologia (SORL) do Hospital de Clínicas de Porto Alegre (HCPA). Forma de estudo: retrospectivo, clínico, não randomizado. Material e método: Foi realizado um estudo retrospectivo dos pacientes que foram submetidos a PAAF no SORL do HCPA entre julho de 1998 e dezembro de 1999. Foram excluídos pacientes ${ }^{1}$ submetidos à PAAF de tireóide ${ }^{2}$ com dados incompletos $^{3}$ que perderam acompanhamento ${ }^{4}$ que não tinham diagnóstico definitivo da lesão. Os resultados das PAAF foram comparados com o diagnóstico definitivo, sendo este estabelecido por exame anatomopatológico (grupo I) ou por evolução clínica e/ou outros exames (grupo II). Resultados: Dos 70 pacientes incluídos no estudo, $7 \%$ apresentaram punções insatisfatórias e, dentre os materiais satisfatórios, houve 88,6\% de acerto diagnóstico. No grupo I encontramos $8 \%$ de punções insatisfatórios e 88,2\% de acerto diagnóstico. Já no grupo II os mesmos dados foram, respectivamente, $6 \%$ e 87,1\%. O índice total de acerto, incluindo as punções insatisfatórias chegou a 81,5\%. Discussão: Os resultados encontrados corroboram os altos índices de acerto diagnóstico das PAAF descritos na literatura, confirmando ser este um método de grande auxílio no diagnóstico de tumorações cervicais. Assim, nos sentimos encorajados a seguir utilizando de forma rotineira tal técnica.

\begin{abstract}
Tntroduction: countless diseases can initially present as a neck lump, what turns its diagnosis not always easy. The fine-needle aspiration biopsy (FNAB) is a simple, safe and effective method in helping the diagnosis of neck lumps. Aim: evaluate the diagnostic performance of FNAB in neck lumps accomplished in the Otorhinolaryngology Department of the Hospital de Clínicas de Porto Alegre (HCPA). Study design: Retrospective, clinical, not randomized. Material and method: a retrospective study of 72 patients submitted to FNAB in SORL of HCPA between July, 1998 and December 1999. Patients $^{1}$ submitted to thyroid $\mathrm{FNAB}^{2}$, with incomplete data ${ }^{3}$ without follow-up and ${ }^{4}$ without definitive diagnosis of the lesion were excluded. The results of FNAB were compared with the definitive diagnosis, established by histopathologic exam (group I) or for clinical evolution or laboratory exams (group II). Results: 70 patients were included in the study, $7 \%$ presented unsatisfactory FNAB and, among the satisfactory materials, there were $88,6 \%$ of correct diagnosis. In group I it was found $8 \%$ of unsatisfactory FNAB and $88,2 \%$ of correct diagnosis, while in group II, $6 \%$ and $87,1 \%$, respectively. The total index of success, including the unsatisfactory FNAB was $81,5 \%$. Discussion: results confirm the high index of success diagnosis of FNAB described in the literature, confirming that this is a helpful method in diagnostic of neck lump. Like this, we are encouraged ourselves to follow using such procedure as a routine.
\end{abstract}

Acadêmico da Faculdade de Medicina da Universidade de Pelotas - RS

Acadêmica da Faculdade de Medicina da Universidade Federal do Rio Grande do Sul

Médica Residente do Serviço de Otorrinolaringologia do Hospital de Clínicas de Porto Alegre

${ }^{4}$ Professor Adjunto do Departamento de Oftalmologia e Otorrinolaringologia da Faculdade de Medicina da Universidade Federal do Rio Grande do Sul Hospital de Clínicas de Porto Alegre - HCPA

Endereço para correspondência: Mariana Magnus Smith

Rua Ramiro Barcelos 2350, Zona 19 / Hospital de Clínicas de Porto Alegre - 90035-003

Telefone: (0xx51) 33168164 / 33439212 - E-mail: mariamagnussmith@hotmail.com

Trabalho apresentado como tema livre no II Congresso Triológico - agosto 2001

Artigo recebido em 08 de novembro de 2001. Artigo aceito em 22 de novembro de 2001 . 


\section{INTRODUÇ̃̃O}

A primeira descrição de retirada de células de tumorações para exame patológico microscópico através de agulhas foi realizada por Kun ${ }^{1}$ em 1847. A partir de então vários autores publicaram métodos diferenciados de punções por agulha de tumorações em diversos locais do organismo ${ }^{2}$. Entretanto, a técnica que conhecemos hoje por punção aspirativa por agulha fina (PAAF) foi detalhadamente descrita e popularizada somente na década de 50 na Escandinávia ${ }^{1,3}$.

Inúmeras são as patologias que podem se apresentar inicialmente como massa cervical e o diagnóstico muitas vezes não é simples de ser obtido apenas levando-se em consideração aspectos clínicos e mesmo após exames de imagem $^{4,5,6}$. É exatamente em lesões cervicais que a PAAF vem demostrando talvez sua maior aplicação, por tratar-se de um método simples, seguro e eficaz no diagnóstico diferencial de tais processos patológicos ${ }^{7,8,9}$.

As indicações de PAAF em lesões cervicais já foram mais restritas, mas hoje grande parte dos autores defende sua prática em todo e qualquer paciente portador de uma tumoração cervical dita "indefinida", ou seja, que não podemos ter confiança absoluta em nosso diagnóstico clínico $^{7,8}$. Vários estudos têm demonstrado, inclusive, sua grande aplicação na população pediátrica ${ }^{10,11}$

No Serviço de Otorrinolaringologia do Hospital de Clínicas de Porto Alegre (HCPA) utilizamos de forma bastante rotineira a PAAF em tumores cervicais e o objetivo deste estudo é determinar o desempenho diagnóstico das punções por nós realizadas.

\section{MATERIAL E MÉTODO}

Foi realizado um estudo retrospectivo com revisão de prontuários médicos de todos os pacientes submetidos a PAAF na região cervical no HCPA entre julho de 1998 a dezembro de 1999. Os critérios de exclusão utilizados foram: 1. Realização de PAAF de glândula tireóidea; 2. Prontuários incompletos; 3. Perda de acompanhamento ambulatorial; 4. Ausência de diagnóstico definitivo da lesão em que foi realizada PAAF.

Os resultados citopatológicos das PAAF realizadas foram comparados com o diagnóstico definitivo da massa puncionada, sendo os pacientes divididos então em dois grupos. Os que foram submetidos à ressecção do tumor e, portanto, apresentavam exame anatomopatológico da peça para comparação diagnóstica, formaram o grupo I. Já nos pacientes do grupo II o diagnóstico definitivo da massa cervical puncionada foi dado pela evolução clínica, por outros exames ou por biópsias realizadas em outros sítios.

\section{Técnica}

As PAAF foram realizadas de acordo com a técnica que rotineiramente utilizamos em nosso serviço, descrita a seguir.
As punções são realizadas no consultório médico, com o paciente sentado na cadeira de exame otorrinolaringológico. A cabeça é voltada para o sentido oposto à lesão, afim de que a massa se torne proeminente à palpação e que um mínimo de tecido mole precise ser perfurado no momento da introdução da agulha.

Realizamos anti-sepsia da pele no local da punção com álcool iodado. Acoplamos uma agulha calibre $21 \mathrm{em}$ uma seringa de $20 \mathrm{ml}$ e introduzimos esta na lesão com nossa mão dominante, enquanto com a outra mão seguramos a massa. Após introduzir a agulha, faz-se pressão negativa na mesma, puxando-se o êmbolo da seringa, e o conjunto então é movido algumas vezes para dentro e para fora da massa, para que as células desprendidas do tumor fiquem dentro da agulha. Após alguns movimentos assim realizados, suspende-se a pressão negativa e, sem o vácuo, retira-se a agulha e é realizado pequeno curativo compressivo no local da punção para evitar a formação de hematomas.

O material coletado é então depositado em lâminas de vidro e com uma lamínula espalhamos o material. Mandamos para o laboratório 4 lâminas: duas a seco para os métodos de Romanovsky e Giemsa e duas em álcool etílico a 95\% para técnica de Papanicolau. O material é identificado e imediatamente levado ao laboratório de citopatologia pela próprio paciente.

Os pacientes retornam em uma semana para receberem o resultado do exame. Todas as coletas com resultados inicialmente insatisfatórios são novamente puncionadas, com a mesma técnica.

\section{RESULTADOS}

Foram inicialmente selecionados 78 pacientes, sendo que 8 foram excluídos ( 2 por perda de seguimento, 1 por ausência de diagnóstico definitivo e 5 por apresentarem prontuários incompletos). Os pacientes com PAAF de tireóide não chegaram a ser estudados por terem sido excluídos diretamente na pesquisa dos prontuários. Dos 70 pacientes, 33 representaram a grupo I (com exame AP subseqüente) e 37 o grupo II (sem exame AP).

No grupo I, 2 (6\%) pacientes tiveram resultado insatisfatório após duas PAAF. Dentre os pacientes com resultado satisfatório, em 87,1\% (27) dos casos o mesmo mostrou-se correto quando comparado com o exame anatomopatológico, restando 12,7\% (4) dos casos com diagnóstico errado. Dentre os erros diagnósticos neste grupo, um foi de falso negativo para células malignas (CP demonstrou adenoma pleomórfico e o AP resultou em carcinoma epidermóide bem diferenciado), outro foi de falso positivo para células malignas (CP com diagnóstico de carcinoma epidermóide e AP de tumor de Warthin) e dois casos de erro de tipo de célula maligna (ambos com diagnóstico citopatológico de carcinoma epidermóide e AP demonstrando linfoma não Hodgkin). 
Já no grupo II em 3 casos a PAAF foi insatisfatória após duas tentativas (8\%). Dentre os 34 pacientes com PAAF satisfatória para análise laboratorial, $30(88,2 \%)$ tiveram diagnóstico correto e nos $4(11,8 \%)$ restantes houve erro diagnóstico. Dos 4 erros, dois foram falsos negativos para células malignas (ambos com CP negativo e que, na evolução clínica do caso, mostrou-se tratar de metástases de carcinoma de cabeça e pescoço) e os outros dois como erro do tipo de célula maligna (ambos com $\mathrm{CP}$ demostrando carcinoma epidermóide e o diagnóstico definitivo sendo de linfomas não Hodgkin, dado por biópsias em outros pontos).

Quando analisamos o grupo total de pacientes (70), o total de PAAF insatisfatórias após duas tentativas foi de $7 \%$. Quando consideramos a primeira tentativa, 18\% dos casos demonstraram material insatisfatório para exame CP. Dentre os resultados satisfatórios, $88,6 \%$ destes foram corretos. Descartando todos os diagnósticos de linfomas, corretos ou não, a taxa de diagnóstico correto sobe para 94,3\%.

Quando analisamos o total de pacientes que realizam PAAF em nosso serviço que resultam em diagnóstico corretos (ou seja, considerando conjuntamente as falhas de material insatisfatório e os erros laboratoriais) chegamos ao percentual de $81,5 \%$.

\section{DISCUSSÃO}

A punção aspirativa por agulha fina foi introduzida em 1847 por Kun. Entretanto, sua prática foi pouco utilizada até as décadas de 40 e 50, especialmente pelos altos índices de complicações descritas, relacionadas ao diâmetro exagerado das agulhas utilizadas na época ${ }^{1}$. A partir de 1950, após ser descrita por um grupo escandinavo, a técnica, hoje considerada clássica, passou a ser mundialmente adotada para investigação de lesões expansivas cervicais ${ }^{3,12}$.

Os índices de complicações são muito baixos, da ordem de menos de $1 \%$ em algumas séries ${ }^{13}$. A PAAF vem sendo realizada com grande sucesso inclusive na população pediátrica $^{1,10,11}$.

Considerando o processo desde a indicação de PAAF e seu resultado citopatológico final, existem dois pontoschave onde podem ocorrer erros: na coleta do material e na avaliação do mesmo na citopatologia.

Quando colocamos "erros de coleta" estamos nos referindo às situações em que nosso material é considerado insatsfatório para análise, ou seja, não há meios de realização de exame citopatológico no mesmo. Tais erros podem ser resultado de algumas falhas já definidas. Em primeiro lugar, como em todo procedimento médico, a técnica de PAAF deve ser respeitada para que haja bons resultados. Parece haver certo consenso na literatura quanto ao uso de seringas de $20 \mathrm{ml}$ e agulhas calibre 21 . Cannon e colaboradores ${ }^{14}$, em 1995, realizaram um estudo quantitativo do material aspirado via PAAF comparando seringas de 5, 10 e $20 \mathrm{ml}$ e agulhas de calibre 21,23 e 27 . A melhor combinação encontrada foi a tradicionalmente utilizada ( $20 \mathrm{ml}$ e calibre 21). É fundamental que seja realizada pressão negativa durante a introdução da seringa na massa e que a mesma seja suspensa antes da retirada. Qualquer material com sangue deve ser desprezado e nova PAAF realizada.

A experiência do coletador também é fator importante nos resultados de PAAF. Jandu e Webster ${ }^{15}$ compararam a acurácia de PAAF realizadas por médicos residentes e por médicos mais experientes e encontraram diferença estatisticamente significativa entre os dois grupos. O uso de métodos de imagem (ultrassonografia ou tomografia computadorizada) para guiar a PAAF melhora a acurácia do método ${ }^{16,17}$, mas estes não são disponíveis em grande escala. Em nosso meio, indicamos a punção guiada por ultrassonografia apenas em massas cervicais profundas, quando não temos a segurança da PAAF realizada a nível de consultório.

Uma vez o material coletado tendo sido satisfatório, as falhas subseqüentes relacionam-se com o exame citopatológico. Em primeiro lugar, é fundamental que o citopatologista tenha experiência em avaliação de material proveniente de $\mathrm{PAAF}^{7,9,18}$. Além disso, de acordo com o tipo de tumor puncionado há maior ou menor probabilidade de acerto diagnóstico. As lesões císticas são de diagnóstico por PAAF mais difícil que os tumores sólidos ${ }^{8}$. A maior dificuldade diagnóstica via PAAF, entretanto, está nas lesões de origem linfoproliferativa ${ }^{19,20}$, pela impossibilidade de definição do tipo celular em muitos casos. Na nossa série apresentada, quando excluímos os pacientes com diagnóstico de linfomas, sejam estes diagnósticos corretos ou não, aumentamos nosso acerto diagnóstico de $88,6 \%$ para 94,3\%.

A percentagem de resultados insatisfatórios descrita na literatura situa-se entre $9,2 \%$ e $12,4 \% \%^{2,4,8,15}$, estando, então, o nosso índice geral de $7 \%$ bastante adequado. Da mesma forma, nossos resultados gerais de acerto diagnóstico por PAAF encontram-se próximos aos dados publicados na literatura pertinente. Fulciniti e colaboradores ${ }^{13}$ encontraram $96,2 \%$ de acurácia em lesões benignas e 86,4\% em lesões malignas, em estudo retrospectivo de 218 PAAF. Carrol e colaboradores avaliaram 78 PAAF realizadas e seguidas de retirada cirúrgica da massa biopsiada com exame anatomopatológico, encontrando acurácia próxima a 95\% $\%^{8}$. Mobley e colaboradores ${ }^{10}$, em 1991, avaliaram 67 PAAF realizadas em crianças e seus resultados foram corretos em 90\% dos casos. Estes são os estudos com acurácia encontrada mais elevada, restando diversos outros com resultados entre $82 \%$ e $89 \% \%^{2,9,16,21}$.

\section{CONCLUSÃO}

O índice de acerto nos diagnósticos citológicos de tumores cervicais por punção aspirativa por agulha fina são elevados, tornando este método arma útil no diagnóstico diferencial de tais lesões. 


\section{REFERÊNCIAS BIBLIOGRÁFICAS}

1. Ramadan H, Wax M, Boyd C - Fine-needle aspiration of head and neck masses in children. Am J Otolaryngol 1997;18(6):400-4.

2. Frable, M.; Frable, W. - Thin-needle aspiration biopsy in the diagnosis of head and neck tumors. Laryngoscope 1974; 84:106976.

3. Frable, M.; Frable, W. - Fine-needle aspiration biopsy revisited. Laryngoscope 1982; 92:1414-18.

4. Bhattacharyya, N. - Predictive factors for neoplasm and malignancy in a neck mass. Arch Otolaryngol Head Neck Surg 1999; 125:3037.

5. Maisel, R. - When your patient complains of a neck mass. Geriatrics 1980; 108:103-8

6. McGuirt, W. - The neck mass. Medical Clin North Am, 83(1):219234.

7. Caminha, G.; Lubianca Neto, J.; Kuhl, G. - Punção aspirativa por agulha fina no diagnóstico de tumores de cabeça e pescoço. Rev Bras Otorrinolaringologia 1996; 62 (3):246-50.

8. Carrol, C.; Nazeer, U.; Timon, C. - The accuracy of fine-needle aspiration biopsy in the diagnosis of head and neck masses. Ir J Med Sc 1998; 167(3):149-51.

9. Mandell, D.; Genden, E.; Bergman, D. et al. - Diagnostic accuracy of fine-needle aspiration and frozen section in nodular thyroid disease. Otolaryngol Head Neck Surg 2001; 124:531-6.

10. Mobley, D.; Wakely, P.; Frable, M. - Fine-needle aspiration biopsy: application to pediatric head and neck masses. Laryngoscope 1991; 101:469-72.

11. Park, Y. - Evaluation of neck masses in children. Am Fam Physician 1995; 51(8):1904-12.

12. Costa, S.S.; Ribeiro, E.; Rollin, G. et al. - Massas cervicais. In Costa, S.S.; Cruz, O.; Oliveira, J.Á. Otorrinolaringologia: princípios e práticas. Ed Artes Médicas, Porto Alegre; 1996; 520-35.
13. Fulciniti, F.; Califano, L.; Zupi, A.; Vetrani, A. - Accuracy of fineneedle aspiration biopsy in head and neck tumors. J Oral Maxillofac Surg 1997; 55(10):1904-7.

14. Cannon, C.; Richardson, L.; Replogle, W.; Halloran, R. - Quantitative evaluation of fine-needle aspiration. Otolaryngol Head Neck Surg 1996; 114(3):407-12.

15. Jandu, M.; Webster, K. - The role of operator experience in fineneedle aspiration cytology of head and neck masses. Int $\mathrm{J}$ Oral Maxillofac Surg 1999; 28(6):441-4.

16. Colnot, D.; Nieuwenhuis, E.; Brekel, M. et al. - Headand neck squamous cell carcinoma: US-guided fine-needle aspiration of sentinel lymph nodes for improved staging. Radiology 2001; 218:289-293

17. Sack, M.; Weber, R.; Weinstein, G. et al. - Image-guided fineneedle aspiration of the head and neck. Arch Otolaryngol Head Neck Surg 1998; 124:1155-61.

18. Reibel, J. - The patient with a neck mass. Comp Ther 1997; 23(11):737-41.

19. Cannon, C.; Richardson, L. - Value of flow cytometry in the evaluation of head and neck fine-needle lymphoid aspirates:a 3year retrospective review of a community-based practice. Otolaryngol Head Neck Surg, 2001; 124:544-8.

20. Daskalopoulou, D.; Tamiolakis, D.; Tsousis, S. et al. - Sources of discrepancies in the diagnosis of Hodgkin's disease by fine-needle aspiration. Arch Anat cytol Path 1996; 44(4):166-73.

21. Akhtar, S.; Huq, I.; Faiz-u-din, M.; Reyes, L. - Efficacy of fineneedle capillary biopsy in the assessment of patiens with superficial lymphadenophaty. Cancer, 81(5):277-80.

22. Alvi, A; Johnson, J - The neck mass. Postg Medicine 1995; 97(5):8797. 